\title{
Sulla connessione delle superficie algebriche reali.
}

\author{
Memoria di Annibale Comessatit (a Padova)
}

Tra i risultati delle mie passate ricerche sulle superficie razionali reali $\left({ }^{1}\right)$, si segnala, quasi a mo' di conclusione, la formula

$$
I+Z=2(\bar{\rho}-1)
$$

che lega l'ordine di connessione totale $Z$ d'una di quelle superficie, cioè della sua pavte reale (composta di una o più falde) al relativo numero base reale $\bar{\rho}$ ed all' invariante $I$ di ZEUTHEN-SEGRE.

Il carattere sintetico della (1), conferitole dal pregio di superare tutte le distinzioni, pur abbastanza complesse, inerenti alla classificazione delle superficie razionali nel campo reale, m'induceva, fin da allora, a prevedere la possibilita di far rientrare quella formula in una relazione più generale valida per lutte le superficie algebriche reali.

Il presente lavoro vuole appunto confermare tal previsione, mostrando che per ogni superficie algebrica reale $F$ della quale con $\rho, \bar{\rho}$ si designino i due numeri base, complesso e reale, e con $R_{2}$ l'ordine di connessione biddmensionale (viferito, ben s'intende, alla riemannimna $V$ ), si ha

$$
Z \leq R_{2}-2(p-\bar{p})
$$

potendosi scrivere l'eguaglianza almeno quando tutti i cicli a due dimensioni della $V$ sono algebrici, cioè $F$ è priva d’ integrali doppî di seconda specie $\left.{ }^{2}\right)$;

(1) Vedi le Memorie, x) Fondamenti per la geometria sopra le superficie razionali dal punto di vista reale [Mathem. Annalen $73(1912)$ pp. 1-72], 3) Sulla connessione delle superficie razionali reali [Questi Annali (3) 23 (1915) pp. 215-284]. La dimostrazione della (1) è in §), \$6.

(1) Ricordiamo che, per una formula di PrCand, il numero $p_{0}$ degl'integrali doppi di $2^{a}$ specie vale $R_{z}-\rho$; quindi se tutti i cicli bidimensionali di $V$ sono algebrici $\left(R_{2}=\rho\right)$ si ha $P_{0}=0$ e viceversa. L'espressione di $p_{0}$ trovasi nel trattato di E. PrCard-G. Srmart, Théonie des fonctions algébriques de deux variables indépendantes [Paris, Gauthier-Villars (1897-1906)], T. $2^{\circ}$, Cap. $12^{\circ}$, n. 18, e, sotto la forma qui 'seritta, nella monografia di S. LeFscheTz, L'Analysis situs et la géometrie algébrique [Paris, Gauthier-Villars (Collec. Borel) (1924)] Nota I, n. 15, alla quale (Cap. IV, segnatamente $\$ \S$ VI, VII) rinviamo anche per quel che riguarda i cicli algebrici. Si osservi poi che quando $P_{0}=0\left(R_{2}=p\right)$ la (2) può scriversi $Z=2 a^{-}-p$, 
in particolare per tutte le superficie di genere geometrico zero $\left({ }^{3}\right)$. Nel caso delle superficie razionali, appena si ricordi che $\rho=R_{z}=I+2\left({ }^{4}\right)$, si vede che la (2) si riduce alla (1).

La conclusione enunciata vien qui raggiunta attraverso ad un' analisi, abbastanza intima, dei rapporti topologici che intercedono tra la riemanniana $V$ di $F$, e la varietá, pure a quattro dimensioni, $W$, immagine delle coppie di punti associati, in $V$, dalla simmetria $S$ che ivi rappresenta il coniugio di $F$. Quest'analisi conduce prima alla relazione, pure notevole

$$
Z=R_{2}-2 h,
$$

nella quale $h$ esprime il massimo di cicli a due dimensioni reali (cioè trasformati in cicli omologhi da $s$, della $V$, tra di loro indipendenti, cioé il numero analogo ad $R_{2}$ per i cicli reali; e dalla (3) si perviene alla (2) precisando il contributo portato al numero $h$ dai cicli algebrici, ch'è appunto $\rho-\bar{\rho}$. Resta cosi precisato anche il significato della relativa deficienza, cioè dell'intero $\tau=h-(\rho-\bar{\rho})$ per cui .

$$
Z=R_{2}-2(\rho-\bar{\rho}+\tau)
$$

e precisamente si vede ch' esso eguaglia il massimo numero di cicli a due dimensioni reali, indipendenti tra di loro e dai cicli algebrici, che posson considerarsi entro alla $V$.

Ulteriori studi intorno a questo carattere $\tau$, potranno condurre a determinare maggiormente la (2). Comunque, è certo che si tratta d'un invariante (assoluto) reale, ciò̀ non esprimibile medianle $i$ soli invarianti complessi della $F$, giacchè due diversi modelli reali (relativi a simmetrie non equivalenti) delle superficie d' una stessa classe complessa, possono dar luogo a valori affatto diversi per $\tau$. É quel che mostro alla fine del lavoro sopra opportuni esempî,

(3) Secondo un teorema fondamentale del Lefscherz (cfi. Ioco cit. (2), Cap. IV, \& VII) i cicli algebrici son caratterizzati dall' annullarsi dei relativi periodi degl'integrali doppî a $i$ prima specie; quindi, se $p_{g}=0$, maneando addirittura tali integrali, tutti $i$ cicli sono algebrici. Non è ancor certo se, viceversa, una superficie priva d'integrali doppî di $2^{a}$ speeie sia anche priva d'integrali doppì di $1^{\mathrm{a}}$ specie $\left(p_{g}=0\right)$, cioè se possano esistere integrali doppî di $1^{\mathrm{a}}$ specie coi periodi tutti nulli (impropri). Cfr. S. LEFsCHETz, On certain numerical invariants of algebraic varieties, with application to abelian varieties, (Prix Bordin, 1919) [Trans. of the Amer. Math. Soc. 22 (1921) pp. 327-482], n. 26; F. SEVERT, Conferencia general sobre la geometria algebraica [Revista Mat. Hispano*Americana (1926) n. 8].

(4) La $R_{2}=I+2$ rientra nella formula generale di PICARd A Alexander, $R_{2}=I+4 q+2$ ( $q$ invegolarità di $F$ ). Cfr. Lefschetz, loco cit. (') Cap. III, n. 10. Per la $p=I+2$ vedi la Mem. cit. (') ß), \$ 6, od anche F. SEven, Sulla totalita delle curve algebriche tracciate sopra una superficie algebrica [Math. Annalen, 62 (1906) pp. 194-223] n. 13. 
che, riferendosi a superficie la cui connessione totale $Z$ è stata determinata direttamente $\left(^{5}\right)$, dànno anche, con altri, un multiplo controllo delle formule ottenute.

Anche in queste ricerche, relative ai problemi di realtà per le superficie algebriche, si rivela la fecondità della teoria della base, dovuta al Severi, specie ora, che, attraverso al LEFsCHETz, sono stati annodati intimi rapporti fra quella teoria e l'analysis situs.

\section{\$ 1. Preliminari sulle riemanniane delle superficie algebriche reali.}

1. Sia $F$ una superficie algebrica reale, appartenente ad uno spazio qualunque, dotata d'un certo numero $m$ (eventualmente nullo) di falde reali $F_{1}, F_{2}, \ldots, F_{n}$.

La distinzione fra le varie falde resta precisata senz' ambiguità, qualunque siano le singolarita di $F$, ricorrendo ad una trasformata reale $F^{\prime}$ di $F$ priva di punti multipli, per cui la distinzione stessa si pone in modo evidente.

Ė pure pressochè evidente la possibilità di sciogliere la singolarità di $F$ mediante una trasformazione birazionale reale. Comunque, ecco come si puó procedere: Supposto dapprima che $F$ non sia razionale nè riferibile a rigata, la si muti anzitutto in una $F^{\prime \prime}$ di $S_{h}$ priva di punti multipli e di curve eccezionali, senza preoccuparsi della realta della trasformazione; e si dica $s$ la simmetria (trasf. antibirazionale involutoria) di $F^{\prime \prime}$, immagine del coniugio di $F$. Il sistema lineare $\left|A+A^{\prime}\right|$ individuato su $F^{\prime \prime}$ da una sezione iperpiana e dalla sua trasformata $A^{\prime}$ mediante $s$, è mutato in sè da s e dotato di curve unite, onde si può riferirlo proiettivamente al sistema degl'iperpiani d'un $S_{k}$ in modo che all'antiproiettività indotta fra $i$ suoi elementi da corrisponda il coniugio di quello spazio $\left({ }^{6}\right)$. Tenendo conto che la s non ha punti fondamentali su $F^{\prime \prime}$ in quanto questa è priva di curve eccezionali $\left({ }^{7}\right)$, si vede che cosi si passa ad una $F^{\prime}$ priva di punti multipli riferita senza eccezioni ad $F^{\prime \prime}$ ed in corrispondenza biràzionale reale con $F$.

(5) In un lavoro, Sulle riemanniane algebriche, in corso di pubblicazione nei Rendiconti del Circolo Matematico di Palermo,

(6) Cfr. loco cit. (1) $x$ ), nn. 8, 12.

(7) Se una trasformazione antibirazionale $s$, d'una superficie $\Phi$ di $S_{h}$ in se stessa, muta un punto $P$ in una curva $\gamma$, questa eccezionale nel senso ordinario della paroln. Difatti se $k$ è il coniugio di $S_{h}$, la superficie coniugata di $\Phi, P$ il punto coniugato di $P$, la $k s$ è una trasformazione birazionale di $\Phi$ in $\Phi$ che muta $P$ in $\gamma$. 
Se la $F$ è razionale o riferibile ad una rigata, non si può escludere sulla $F^{\prime \prime}$ priva di punti multipli l'esistenza di punti fondamentali per $s$; ma essi si potranno far sparire ad uno ad uno trasformando $F^{\prime \prime}$ mediante il sistema di tutte le forme d'ordine abbastanza alto passanti per il punto che si considera, e dopo ciò si sarà ricondotti al caso precedente $\left(^{8}\right)$.

Indicheremo con $Z_{1}, Z_{2}, \ldots, Z_{m}$ gli ordini di connessione delle falde $F_{1}$, $F_{2}, \ldots, F_{m}$ di $F$. Il numero $Z=\sum_{i=1}^{m} Z_{i}-2 m+-2$ si dirà ordine di connessione totale della superficie $\left({ }^{9}\right)$.

Per fissare senz' ambiguità i valori delle $Z_{i}$ giova anche qui il riferimento alla $F^{\prime \prime}$, purchè su questa si ripristinino le curve eccezionali (di $1^{*}$ specie) della $F$. La possibilita di tal ripristino mediante una trasformazione birazionale reale che non introduca singolarità è manifesta, dal momento che su $F$ quelle curve son reali o a due a due coniugate e quindi devon dedursi da punti reali o da coppie di punti coniugati della $F^{\prime}\left({ }^{10}\right)$.

D' altra parte quel ripristino è necessario perchè la comnessione delle falde di $F^{\prime}$ sia fissata in modo confacente alla $F$ e perché resti individuata senza ambiguità la connessione bidimensionale della riemanniana $V$ che andiamo a considerare $\left({ }^{11}\right)$.

La superficie $F^{\prime}$ cosi modificata l'indicherá con $\Phi$, e le sue falde con $\Phi_{1}$, $\Phi_{2}, \ldots, \Phi_{m}$

2. Trasportiamoci ora alla riemanniana $V$ di $F$ ch'è ormai definita topologicamente in modo preciso, come l'ente i cui elementi (punti) sono in corrispondenza biunivoca, continua, e senza eccezioni, coi punti reali e complessi

(8) Avvertasi che $F$ può possedere linee o punti isolati reali, ma in ogni caso multipli e di molteplicitâ pari; perơ in senso invariantivo quej punti o i punti di quelle linee non devon considerarsi come reali, in quanto spariscono sulla $F^{\prime \prime}$.

(9) Cfr. loco cit. (1) B) n. 8. Notiamo che la nostra definizione per l'ordine di connessione d' una falda è in pieno accordo con quella generale del Lefschetz (loco cit. ( ${ }^{2}$ ), Cap. I, n. 6) relativa agli ordini di connessione di varietà qualunque, ai quali si riferisce la formula $d i$ Eulero generalizzata (ibid., n. 8) sotto la forma che poi dovremo applicare.

(10) Il caso delle superficie razionali o riferibili a rigate domanderebbe qualche complemento su cui crediamo di poter sorvolare. Ad ogni modo il primo è esaurientemente considerato al $\mathrm{n} .13$ della Mem, $\beta$ ) citata in $\left(^{2}\right)$.

(14) Si ricordi (loco cit. (1) ß), \$3) che mutando un punto d'una falda in una curva ecce. zionale se ne altera la connessione e che la $V$ non è individuata di fronte alla relazione d'omeomorfismo se non quando su $F$ è fissato il regime delle curve eccezionali (quindi gl' in. varianti relativi). 
di $\Phi$. Al coniugio di $\Phi$ (e di $F$ ) corrisponde in $V$ una simmetria $S$, cioè una trasformazione involutoria, dappertutto biuni voca e priva di punti fondamentali, che possiede $m$ superficie $V_{1}, V_{2}, \ldots, V_{m}$ luogo di punti uniti, immagini delle falde $\Phi_{i}$, quindi omeomorfe ad esse, omogenee, chiuse, prive di singolarità e di punti comuni.

Notoriamente la $V$ è bilatera, quindi possiamo supporla orientata, fissandone la faccia posiliva, o, ciò ch'è lo stessò, il verso positivo d' un' indicatrice (quindi di tutte le altre). Ricordiamo che per indicatrice orientata relativa ad un punto $P$ s'intende una piccola cellula (pentaedro) contenente nel suo interno $P$, per $\mathrm{i}$ cui vertici sia fissata la classe delle permulazioni positive.

Se $P^{\prime}$ è il punto di $V$ associato a $P$ da $S$, ad una indicatrice positiva di $P$ resta associata $u n^{\prime}$ indicatrice relativa a $P^{\prime}$ che puó avere il verso positivo o l'opposto. Vogliam far vedere che, contrariamente a quel che ha luogo sulle superficie riemanniane, la nuova indicatrice è ancor positiva, cioè che la simmetria $\mathrm{S}$ è una trasformazione direlta per la varietà $\mathrm{V}$.

La divergenza fra i due casi bi-e quadri-dimensionale si rivela chiaramente negli esempî elementari relativi alle riemanniane della retta $z(=x+i y)$ e del piano $z, w(w=u+i v)$. Nel primo caso si puó assumere come riemanniana il piano $x y$ (non disturbando l'eccezione all'infinito) ove il coniugio di $z$ è rappresentato dalla simmetrial $y^{\prime}=-y$ rispetto all' asse $x$, nel secondo lo $S_{4}$ reale $x, y, u, v$, ove si ha amalogamente la simmetria $y^{\prime}=-y, v^{\prime}=-v$ rispetto al piano $y=v=0$. Due indicatrici (triangoli, risp. pentaedri) associate sono, nel primo caso, contraverse, nel secondo equiverse, perchè $\mathbf{i}$ determinanti

$$
\begin{array}{rr}
\left|x_{i}, y_{i}, 1\right|\left|x_{i},-y_{i}, 1\right| & (i=1,2,3) \\
\left|x_{i}, y_{i}, u_{i}, v_{i}, 1\right|\left|x_{i},-y_{i}, u_{i},-v_{i}, 1\right| & (i=1,2,3,4,5)
\end{array}
$$

hanno segno contrario nell' uno, segno eguale nell'altro.

Quando la $F$ ha punti reali, si puó sempre ricondursi al caso del piano, bastando limitarsi a considerare l'effetto di $S$ sui punti prossimi ad un punto unito, ed osservare che l'intorno complesso d'un generico punto reale di $F$ puó riferirsi biunivocamente all' analogo intorno d' un punto reale d' un piano $\pi$ per proiezione da un punto reale 0 . Oppure si puó osservare che, in $V$, la $S$ nell'intorno d'un punto (unito) d' una delle superficie $V_{i}$ ha il carattere d'un'omografia involutoria di $s_{4}$, quindi, attesa la dimensione di $V_{i}$, d' una simmelria rispetto ad un piano, che, come s'è visto, è una trasformazione direlta. 
Ad esuberanac valga infine la segueite dimostrazione, indipendente dall'ipotesi che $F$ contenga punti reali:

Si fissino su $F$, com' è sempre possibile, due curve algebriche reali $C, D$ che abbiano due fra le intersezioni $p, p^{\prime}$ immaginarie coniugate, ad esempio le intersezioni di $F$ con due forme reali del relativo spazio opportunamente scelte; e siano $\gamma, \delta$ i corrispondenti cicli a due dimensioni di $V$ segantisi nei punti $P, P^{\prime}$ associati in $S$. Per un teorema di LEFscherz, orientati opportunamente $\gamma, \delta$, il numero effettivo (aritmetico) delle loro intersezioni coincide col numero algebrico $(\gamma, \delta)$ di Kroneckel-Poncaré $\left({ }^{12}\right)$, onde ciascumo dei punti $P, P^{\prime}$ porterà quest' ultimo contributo +1 . Pertanto se $P Q R, P S T$, son due indicatrici positive di $\gamma, \delta$, sarà $P Q R S T$ un' indicatrice positiva della $V\left({ }^{13}\right)$.

Ma sul ciclo $\gamma$, ch'è riemanniana di $C$, la simmetria indotta da $S$ (immagine del coniugio di $C$ ) è una trasformazione inversa, quindi detti $Q^{\prime}, R^{\prime}$ i corrispondenti di $Q, R$, sarà $P^{\prime} R^{\prime} Q^{\prime}$ un'indicatrice positiva di $\gamma$ ed analogamente $P^{\prime} T^{\prime} S^{\prime}$ un'indicatrice positiva di $\delta$; onde per il fatto che anche $P^{\prime}$ porta a $(\gamma, \delta)$ contributo +1 , la $P^{\prime} R^{\prime} Q^{\prime} T^{\prime \prime} S^{\prime}$ sarà un'indicatrice positiva di $V$. Dopo ciò basta osservare che la permutazione $P^{\prime} R^{\prime} Q^{\prime} T^{\prime} S^{\prime}$ è della stessa classe della $P^{\prime} Q^{\prime} R^{\prime} S^{\prime} T^{\prime}$ relativa all'indicatrice trasformata di $P^{\prime} Q R S T$ mediante $S$ per concludere che anche quell'indicatrice è positiva per $V$, c. d. d.

\section{§ 2. Rappresentazione della riemanniana simmetrica $V$, sopra una varietà doppia $W$. Relazione fra $i$ cinque ordini di connessione $\boldsymbol{r}_{1}, \boldsymbol{r}_{2}, \boldsymbol{R}_{1}, \boldsymbol{R}_{2}, Z$.}

3. Indicheremo con $W$ l'ente a quattro dimensioni i cui elementi (punti) rappresentano senza eccezioni le coppie dei punti di $V$ associati in $S$. La varietà $W$ è topologicamente ben definita, e non ha interesse fissarne un qualsiasi modello.

Le varieta $W, V$ son legate da una corrispondenza $(1,2), T$, che ha su $W$ come elementi di diramazione $m$ falde $W_{i}$ riferite biunivocamente senza eccezioni alle $V_{i}$ (ed alle falde $F_{i}$ di $F$ ) quindi omeomorfe ad esse, prive di punti multipli e di punti comuni, ecc.

Proviamo che anche la varietà $W$ è bilatera ${ }^{(4)}$.

(12) Lefschetz, loco cit. (2), Cap. 11, n. 6 .

(13) Ibid., Cap. I, n. 12.

(14) Invece nell'analogo caso relativo alle curve, è noto da KLEIN e Werchoto che la superficie $W$ è unilatera o bilatera. a seconda che $V$ (colla simmetria $S$ ) è diasimmetrica od ortosimimetrica. Cfr. F. KLeIN, Riemann'sche Flächen [Leipzig, Tenbner (1906) Parte $3^{a}$, I, B, 
Sia $P$ un punto di $W$, immagine della coppia $P_{1}, P_{2}$. di $V, \sigma$ un cammino chiuso qualunque di $W$ che parta da $P$ e vi ritorni, $J$ un'indicatrice (orientala) di $W$ relativa a $P$. Si tratta di far vedere che, quando un punto $Q$ di $W$ descrive $\sigma$ in un verso a partire da $P$ portando seco la $J$, quell'indicatrice nou puó mai, al ritorno, risultare invertita.

Alla $J$ corrispondono in $V$ due indicatrici $J_{1}, J_{2}$, relative a $P_{1}, P_{2}$ e trasformate l'una nell' altra da $S$. Poichè $S$ é diretta, le $J_{1}, J_{2}$, saramo equiverse, ad es. entrambe posilive per $V$.

Ció premesso, e supposto inoltre, com'è lecito a meno d'una piccola deformazione, che $\sigma$ non incontri le $W_{i}$ (del resto tale ipotesi non è essenziale) posson darsi due casi:

a) Al ritorno di $Q$ in $P$ i due punti corrispondenti $Q_{1}, Q_{2}$, che partono da $P_{1}, P_{2}$ ritornano pure alle posizioni iniziali; allora a $\sigma$ corrispondono su $V$ due cammini $\sigma_{1}, \sigma_{z}$, distinti, e privi di punti comuni, l'uno descritto da $Q_{1}$, l'altro da $Q_{2}$. Quando $Q_{1}$ descrive $\sigma_{1}$, l'indicatrice $J_{1}$ associata ad $J$ ritorna in $P_{t}$ col verso iniziale perchè $V$ è bilatera; dunque lo stesso accade di $J$ al ritorno in $P$.

b) I due punti $P_{1}, P_{2}$ ritornano scambiati tra di loro; allora mentre $Q$ descrive $\sigma, Q_{1}$, descrive un cammino aperto $\sigma_{1}$ coll' origine in $P_{1}$ e l'estremo in $P_{2}$. Durante il percorso l'indicatrice $J_{1}$ relativa a $Q_{1}$ ed associata ad $J$ resta sempre positiva per $V$, dunque all'arrivo in $P_{2}$, si trova concorde con $J_{2}$; e ciò viene a dire che $J$ al ritomo in $P$ ha ancora il verso di partenza.

Infine è ovvio che anche la $W$ é chiusa, dal momento che le $W_{i}$ essendo superficie, non possono produrvi frontiere.

4. Immaginiamo ora tracciata su ciascuna $W_{1}$ una triangolazione $\Delta_{i}$ con $\alpha_{0}^{(i)}$ vertici, $\alpha_{1}^{(i)}$ lati, $\alpha_{2}^{(i)}$ facce, e ricordiano che per la formula di Eulero a cui può affidirsi (anche se $W_{i}$ è unilatera) la definizione di $Z_{i}$ si ha

$$
\alpha_{0}^{(i)}-\alpha_{1}^{(i)}+\alpha_{2}^{(i)}=2-Z_{i} .
$$

Fissiamo poi auche in $W$ una triangolazione $\Delta$, cioè una decomposizione in cellule (senza punti interni comuni, ecc.) che conlenga le $\Delta_{i}$, cioè abbia fra i suoi vertici, lati, facce a due dimensioni, quelli delle $\Delta_{i}$ ed indichiamo con $a_{0}, a_{1}, \ldots, a_{4}$ i numeri dei relativi elementi delle diverse dimensioni.

nn. 3.5̃; G. WexchoLd, Ueber' symmetrische Riemann'sche Flächen und die Periodicitätsmo. duln, ecc. [Zeitsch. fuir Math. u Phys. 28 (1883), pp. 321-351] \$\$ 3.4. Per la relazione tra $V$ e $W$ nel caso delle curve vedi anche il lavoro (5. $n$. 5 . 
Ad una cellula $K$ della triangolazione $\Delta$ corrispondono in $V$ due cellule $K_{1}, K_{2}$, che, come si vede subito tenendo conto della comnessione semplice di $K$, non hanno punti inlerni comuni. La sorrapposizione puó presentarsi soltanto per elementi di frontiera di dimensione non superiore a due, e precisamente si verifica per quegli elementi che corrispondono agli (eventuali) elementi di $K$ appartenenti alle $\Delta_{i}$.

Si vede cosi che $\Delta$ induce in $V$ un' analoga triangolazione $\Delta^{\prime}$, e poichè nel passaggio di $W$ a $V$ ciascun elemento si sdoppia, meno quelli che appartengono alle $\Delta_{i}$, ne viene, che, indicandosi con $A_{i}$ i numeri analoghi agli $a_{i}$ per la $\Delta^{\prime}$ sarà

$$
\begin{gathered}
A_{1}=2 a_{4}, \quad A_{3}=2 a_{3}, \quad A_{2}=2 a_{2}-\sum_{i=1}^{m} \alpha_{2}^{(i)}, A_{1}=2 a_{1}-\sum_{i=1}^{m} \alpha_{1}^{(i)}, \\
A_{1}=2 a-\sum_{i=1}^{m} \alpha_{0}^{(i)} .
\end{gathered}
$$

Se ora s'introducono gli ordini di connessione $R_{i}\left(i=0,1, \ldots, 4 ; R_{0}=R_{4}=1\right.$, $R_{1}=R_{3}$ ) delle diverse dimensioni, relativi a $V$, e gli analoghi $r_{i}$ relativi a $W$, e si combinano le relazioni

$$
\sum_{h=0}^{4}(-1)^{h} A_{h}=\sum_{h=0}^{4}(-1)^{h} R_{h}, \quad \sum_{h=0}^{4}(-1)^{h}\left(I_{h}=\underset{h=0}{\sum}(-) 1^{h} v_{h},\right.
$$

scritte a norma della formula di Eulero generalizzata $\left({ }^{15}\right)$, colle precedenti (5) (6) e colla formula

$$
Z=\sum_{i=1}^{m} Z_{i}-2 m+2
$$

che esprime l'ordine di connessione totale di $F$ in funzione degli $Z_{b}$, si ottiene la relazione fondamentale

$$
Z=R_{2}-2 r_{2}-2\left(R_{1}-2 v_{1}\right)\left({ }^{16}\right)
$$

(15) Cfr. Lefschetz, loco cit. ("), Cap. I, n. 8.

('6) Se le $W_{i}$ mancano, cioè se $F^{\prime}$ non ha punti reali, le $x^{(i)}$ della (5) devon porsi eguali allo zero, ed allora si vede che la (9) sussiste ancora purchè si ponga $Z=2$. Circa l'oppor* tunità di attribuire convenzionalmente ordine di connessione 2 alle superficie per cosi dire inesistenti (topologicamente) vedi la nota $\left({ }^{(5)}\right)$ del lavoro citato in $\left({ }^{5}\right)$. 


\section{§ 3. Confronto tra le connessioni di $r, W$. Relazione $\boldsymbol{R}_{1}=2 r_{1}$ tra le connessioni lineari, e prima forma della conclu- sione.}

5. Il secondo membro della (9) dipende oltre che dai caratteri topologici $R_{1}, R_{2}$ della $V$, notoriamente esprimibili mediante gli invarianti (assoluti e relativi) di $F$, anche dagl'incogniti caratteri $r_{1}, r_{2}$ della $W$. Si pone pertanto il problema di eliminare tali caratteri in base ad un'ulteriore analisi dei rapporti topologici tra le due varietà $V, W$.

Sia $C$ un ciclo, p. es. lineare della varietá $V$. Esso potrà risultare dal l'unsieme di più parti, anche contate piu volte; ma in ogni casu mediante una piccola deformazione e l'aggiunta di segmenti percorsi nei due sensi, che poi si scinderanno ciascuno in due archi distinti, si potrà ridurre $C$ ad un unico continuo orientato, tutto costituito da punti semplici. Analogamente per i cicli a due dimensioni, salvo l'eventuale presenza d' un gruppo discreto di punti in cui il ciclo attraversa se stesso, che non porta alcun pregiudizio al seguito.

Sempre a meno d'una piccola deformazione si potrà supporre che $C$ non abbia punti commini (o ne abbia un gruppo discreto se è a due dimensioni) col suo corrispondente $C^{\prime}$ secondo $S$. In tali condizioni è ovvio che l'insieme dei punti di $W$ omologhi (in $T^{-1}$ ) a quelli di $C$, è un ciclo $\gamma$ (orientato) di $W$ i cui punti sono in corrispondenza biunivoca con quelli di $C$ e di $C^{\prime}$. Si dirà che $\gamma$ è il ciclo corrispondente a $C$, oppure a $C^{\prime}$; ed è pure ovvio che se ai cicli $C, D$ corrispondono $\gamma$, $\delta$, a $C+D$ corrisponde $\gamma+\delta$, ecc.

Partiamoci invece da un ciclo $\gamma$ di $W$, ridotto, come prima $C$, ad un unico continuo orientato, ecc., e consideriamo in $V$ la figura $\Gamma$ costituita dai punti $P_{1}, P_{2}$ omologhi dei punti $P$ di $\gamma$. E chiaro che possono darsi due casi:

a) Al variare di $P$ su $\gamma$ i due punti $P_{1}, P_{2}$ descrivono due continui distinti ; allora questi son due cicli $C, C^{\prime}$ di $V$ associati in $S$ e riferiti (ciascuno) biunivocamente a $\gamma$ come sopra;

b) I punti $P_{1}, P_{2}$ descrivono un unico continuo $\Gamma$ in corrispondenza $(2,1)$ con $\gamma$ e trasformato in sè da $S$. Allora la biunivocità della corrispondenza puó ristabilirsi contando due volte ciascun punlo $d i \gamma, 0$, meglio ancora, procedendo come segue: Con una piccola deformazione si muti $\Gamma$ in un ciclo $\Gamma_{\text {, }}$ distinto dal corrispondente $\Gamma_{1}^{\prime}$ (in $S$ ) e si dica $\gamma_{1}$ il ciclo corrispondente di $W$. Si vede allora subito che nel passaggio da $\gamma$ a $\gamma_{1}$ ciascun punto di $\gamma$ si sdoppin, restando le due parti (archi od aree) di $\gamma_{1}$ prossime ad una parte di $\gamma$ concor. 
demente orientate; sicchè in definitiva si può dire che al ciclo $\Gamma_{1}, 0$, il che è lo stesso, al suo omologo $\Gamma$, corrisponde in $W$ il ciclo $2 \gamma\left({ }^{17}\right)$.

Riassumendo, si ha che ogni ciclo $C$ di. $V$ (assieme al suo associato $C^{\prime}$ ) ha un determinato corrispondente $\gamma$ in $W$; inversamente, un $\gamma$ di $W$ puó non avere corrispondente in $V$, ma lo ha certo $2 \gamma$.

6. Siano $C$ e $\gamma$ corrispoudenti nel senso ora chiarito, $C^{\prime}$ l'associato di $C$. Premesso che per esprimere la relazione "omologo $a$ » faremo uso del segno $=$ dimostriamo i seguenti lemmi:

A) Se $C=0$, anche $\gamma=0$;

B) Se $\gamma=0, C+C^{\prime}=0$.

Ragioneremo per semplicità nel caso dei cicli linear $i$.

A) Se $C=0$, esiste una superficie bilater $a$ aperta $\mathbf{\Omega}$ di cui $C$ costituisce la frontiera. Ad $\Omega$ corrisponde in $W$ una superficie $\omega$ pure bilatera riferita biunivocamente ad $\boldsymbol{\Omega}$ (potendosi supporre che $\Omega$ sia distinta da $\boldsymbol{\Omega}^{\prime}$ ed abbia con essa in comune solo un gruppo discreto di punti) ed avente per orlo $\gamma$; dunque $\gamma=0$.

B) Se $\gamma=0$, si ha aualogamente in $W$ una superficie bilatera aperta $\omega$, che potremo raffigurarci intuitivamente sotto la forma d' un disco avente per orlo $\gamma$. Considerando per ogni punto $P$ di $\omega$ i due punti corrispondenti $P_{1}, P_{2}$ di $V$ posson darsi due casi :

$\left.b_{1}\right)$ Al variare di $P$ su $\omega, P_{1}, P_{2}$ descrivono due continui distinti. Allora clascuno di essi $\dot{\epsilon}$ un disco, omeomorfo ad $\omega$ avente per orlo $C, 0 C^{\prime}$; dunque $C=0, C^{\prime}=0$, ed infine $C+C^{\prime}=0$.

$b_{2}$ ) Al variare di $P$ su $h, P_{1}, P_{2}$ descrivono un unico continuo $\Omega$. Allora $\Omega$ è uua superficie bilatera, della forma d' un tubo avente per orlo $C+C^{\prime}$; dunque $C+C^{\prime}=0$.

Il carattere bilatero di $Q$ si mette in evidenza facilmente, notando che, orientata la $\omega$, in ogni punto $P_{1}$ di $\Omega$ è definita un'indicatrice positiva che è quella corrispondente all'indicatrice positiva $J$ di $\omega$ nel punto $P$ corrispondente a $P_{4}$. Nè il caso che $P_{\mathfrak{a}}$ coincida con $P_{2}$, cioè che $P$ sia una delle eventuali intersezioni (isolate) di $\omega$ colle $W_{i}$ dà luogo ad ambiguità, per quanto ad $J$ corrispondano due indicatrici $J_{1}, J_{2}$ relative a $P_{1}=P_{2}$; si tratta invero d'indicatrici concordi perchè la $S$ ha, su $Q$, nell' intorno di $P_{1}=P_{2}$ carallere divetto, non essendovi divezioni unite per $P_{1}$.

(17) Per gli scopi finali, se $C$ e $\gamma$ son corrispondenti, è indifferente sostituirli con cicli omologhi. Ma nelle considerazioni del prossimo numero giova supporre che $C$ e $\gamma$ siano effettivamente corrispondenti, cioè associati dalla $T$. 
Importa poi precisar bene che la frontiera completa di $\mathrm{\Omega} e \mathrm{C}+\mathrm{C}^{\prime}$, non $\mathrm{C}-\mathrm{C}^{\prime}$, intendendosi che si parli di frontiera or ientata $\left({ }^{18}\right)$, altrimenti la questione non avrebbe senso. Ora se l'orientazione di $\omega$ è fissata in modo che $\gamma$ (col suo verso) ne sia frontiera orientata, detta $B C$ un' indicatrice positiva di $\gamma$ ed $A$ un punto interno ad $\omega$ e prossimo a $B, C$ sarà $A B C$ un'indicatrice positiva di $\omega$; quindi col solito significato dei simboli saranno $A_{1} B_{1} C_{1}$, $A_{2} B_{2} C_{2}$ indicatrici positive di $\mathbf{Q}$ aderenti $a \mathrm{C}, \mathrm{C}^{\prime}$. Ma d'altra parte $B_{1} C_{1}, B_{2} C_{2}$ sono indicatrici positive di $C, C^{\prime}$ dunque la frontiera orientata di $\Omega$ è proprio $C+C^{\prime}$ come asserito.

7. Consideriamo ora due ordini di connessione associati (ciò del medesimo indice) di $V, W$, che, sopprimendo provvisoriamente gl'indici, indicheremo con $R, r$ e proviamo anzitutto che $r \leq R$.

Fissiamo allo scopo, in $V, R$ cicli indipendenti $C_{1}, C_{2}, \ldots, C_{R}$ della dimensione desiderata, ed attribuiamo ai simboli $\gamma_{i}, C_{i}^{\prime}$ il solito significato. A prova dell'asserto mostreremo che ogni ciclo $\delta$ di $W$ è dipendente da $\gamma_{1}, \gamma_{2}, \ldots, \gamma_{R}$.

Difatti, se non $\delta$, certo $2 \delta$ è il corrispondente d'un ciclo $D$ di $V ;$ e poichè i $C_{i}$ dànno una base su $V$, cosi si avrà un' omologia

$$
\mu D+\lambda_{1} C_{1}+\lambda_{2} C_{2}+\ldots+\lambda_{R} C_{R}=0,
$$

dalla quale per il lemma $A$ segue la

$$
2 \mu \delta+\lambda_{1} \gamma_{1}+\lambda_{2} \gamma_{2}+\ldots+\lambda_{R} \gamma_{R}=0
$$

che esprime quanto asserito.

Dopo ciò è chiaro che il valore di $r$ sarebbe pienamente determinato se lo fosse il numero delle omologie indipendenti che legano i cicli $\gamma_{1}, \gamma_{2}, \ldots, \gamma_{R}$. Ora si vede che se ad̀ esempio $C_{i}^{\prime}=-C_{i}$, cioè se $C_{i}+C_{i}^{\prime}=0$, una di tali omologie, è, per il lemma $A, \gamma_{i}+\gamma_{i}=0$, cioè $2 \gamma_{i}=0$, e ciò suggerisce il seguente precedimento che conduce a determinare, almeno in un certo senso, il valore cercato, in quanto ne mette in vista un significato importante:

Si considerino gli $R$ cicli $C_{i}+C_{i}^{\prime}$, che la $S$ muta in se stessi (in cicli omologhi) e che perció, seguendo una terminologia introdotta altrove, diremo cicli reali $\left({ }^{19}\right)$, e si supponga che fra essi intercedano $R-h$ e non più omologie

(18) Cfr. Lefschetz, loco cit. (9), Cap. I, nn. 3, 4, ed anche O. VEBLEN, Analysis Situs (The Cambridge colloquium 1916) [Publ. by the Amer. Math. Soc., New York (1922)], Cap. IV, nn. 10, 25.

(19) Vedi la prima delle Memorie, Sulle varietà abeliane reali. [Questi Annali (4) 2 (1924-25), pp. 67-106, е 3 (1925-26), pp. 27-71], n. 3. 
indipendenti, di guisa che, sceltine opportunamente $h$, siano ad esempio i primi, tutti gli altri sian dipendenti da essi. Posto $A_{i}=C_{i}+C_{i}^{\prime}(i=1,2, \ldots, h)$ è facile vedere che $A_{1}, A_{2}, \ldots, A_{h}$ dànno una base per $i$ cicli reali di $V$, cioè che ogni ciclo reale di quella riemanniana dipende dagli $A_{i}$.

Ed invero se $D$ è un tal ciclo, legato ai $C_{i}$ dalla (10), trasformando mediante $S$ e tenendo conto che $D=D^{\prime}$ si avrà anche

$$
\mu D+\lambda_{1} C_{1}^{\prime}+\lambda_{2} C_{2}^{\prime}+\ldots+\lambda_{R} C_{R}^{\prime}=0,
$$

quindi sommando

$$
2 \mu D+\lambda_{1}\left(C_{1}+C_{1}{ }^{\prime}\right)+\lambda_{2}\left(C_{2}+C_{2}{ }^{\prime}\right)+\ldots+\lambda_{R}\left(C_{R}+C_{R}{ }^{\prime}\right)=0
$$

da cui, ricordando che tutti i $C_{i}+O_{i}$ dipendono dagli $A_{i}$, segue subito l'asserto.

Indichiamo con $\alpha_{1}, \alpha_{2}, \ldots, \alpha_{n}$ i cicli di $W$ corrispondenti agli $A_{i}$. Vogliam provare che tali cicli dànno una base in W, cioè che $r=h$.

Intanto gli $\alpha_{i}$ sono indipendenti, perchè se fosse

$$
\lambda_{1} \alpha_{1}+\lambda_{2} \alpha_{2}+\ldots+\lambda_{h} \alpha_{n}=0,
$$

risulterebbe per il lemma $B$

$$
\lambda_{1}\left(A_{1}+A_{1}^{\prime}\right)+\lambda_{2}\left(A_{2}+A_{2}{ }^{\prime}\right)+\ldots+\lambda_{h}\left(A_{h}+A_{h}{ }^{\prime}\right)=0,
$$

cioè, tenuto conto che gli $A_{i}$ son reali e quindi $A_{i}^{\prime}=A_{i}$

$$
2 \lambda_{1} A_{1}+2 \lambda_{2} A_{2}+\ldots+2 \lambda_{h} A_{h}=0,
$$

incompatibile coll' indipendenza degli $A_{i}$.

Sia poi $\beta$ un ciclo qualunque di $W$. Se non $\beta$, certo $2 \beta$ è il corrispondente d'un ciclo $B$ di $V$, e poichè $B+B^{\prime}$ è un ciclo reale quindi dipendente dagli $A_{i}$, cosi sussisterà un' omologia

$$
\mu\left(B+B^{\prime}\right)+\lambda_{1} A_{1}+\lambda_{2} A_{2}+\ldots+\lambda_{h} A_{h}=0 .
$$

(con $\mu \neq 0$ ) dalla quale per il lemma $A$ (e ricordando che anche a $B^{\prime}$ corrisponde $2 \beta$ ) segue

$$
4 \mu \beta+\lambda_{1} \alpha_{1}+\lambda_{2} \alpha_{2}+\ldots+\lambda_{h} \alpha_{h}=0,
$$

col risultato che ogni ciclo $\beta$ di W dipende dagli $\alpha_{i}$. Ricordando il significato di $h$ si ha quindi in definitiva che:

$L^{\prime}$ ordine di connessione $\gamma^{*}$ della varietà $W \dot{e}$ eguale al massimo numero di cicli reali indipendenti, della relativa dimensione, che appartengono alla riemanniana $V$. 
8. Non è consentito proceder oltre senza separare i due casi fin qui accomunati, facendo appello a proprieta di carattere più specifico. Rivolgendoci dapprima alla connessione lineare, per cui le circostanze si presentano più favorevoli, posto $R_{1}=2 q$ ( $q$ irregolarità di $F$ ) mostriamo che $h=\nu_{1}=q=\frac{R_{4}}{2}$.

Perciò fissiamo in $F, q$ integrali picardiani di $1^{\text {a }}$ specie $u_{1}, u_{2}, \ldots, u_{q}$ indipendenti e reali, e ricordiamo che i periodi d'uno di essi, presi lungo due cicli $C, C^{\prime}$ associati in $S$ sono coniugati, quindi quelli relativi ai cicli reali sono reali $\left({ }^{20}\right)$. Inoltre aggreghiamo ad $A_{1}, A_{2}, \ldots, A_{h}$ altri $R_{1}-h=2 q-h$ cicli $B_{1}$, $B_{2}, \ldots, B_{q q-h}$, in modo da formare una base per i cicli lineari di $V$.

Basta ora ragionare cosi : Se fosse $h>q$, quindi $2 q-h<q$, dai periodi d'una opportuna combinazione lineare a coefficieuti reali $u$ degli $u_{i}$, relativi ai cicli $B_{j}$, si potrebbero far sparire le parti immaginarie; e cosi si otterrebbe un integrale coi periodi tutti reali. Se poi fosse $h<q$ si potrebbe far si che i periodi di $u$ relativi agli $A_{i}$, e quindi a tutti i cicli reali di $V$ fossero nulli; ma allora, qualunque fosse il ciclo $C$, sarebbe nullo il periodo di $u$ relativo a $C+C^{\prime}$, quindi quello lungo $C$ immaginario puro, ed in definitiva sarebbero immaginarî puri tutti $i$ periodi di $u\left({ }^{21}\right)$. In entrambi i casi si cade in assurdo.

In forza della relazione $R_{1}=2 v_{1}$ l'espressione entro parentesi a secondo membro della (9) è nulla, onde, posto $r_{2}=h$, si ha col significato stabilito per quel carattere

$$
Z=R_{2}-2 h
$$

\section{§ 4. Intervento dei numeri base e loro contributo alla con- nessione bidimensionale $h=r_{2}$ di $W$. Seconda forma della conclusione.}

9. A. formare l'insieme dei cicli a due dimensioni reali di $V$, contribuisce, parzialmente o totalmente, la classe dei cicli algebrici. Ci proponiamo di dimostrare che $i l$ contributo di questa classe al numero $h$ é espresso da $\rho-\bar{\rho}$, cioè che in $V$ si possono trovare $\rho-\bar{\rho}$ e non più cicli algebrici reali fra di loro indipendenti. Ne conseguirà che $h \geq \rho-\bar{\rho}$, e, posto $h=\rho-\bar{p}+\tau$ si avranno le (2) (4) della prefazione coll' avvertito significato di $\tau$.

Premettiamo alcune osservazioni sui cicli algebrici della riemanniana $V \mathrm{e}$ sui loro rapporti colle curve tracciate sulla superficie $F$.

(20) Vedi loco cit. $\left({ }^{19}\right)$, Mem. $1^{\mathrm{a}}$, nn, 1-3.

(ij) $\mathrm{Si}$ è. ripetuto in sostanza il ragionamento della succitata Memoria, n. 2, e, con qualche aggiunta, si potrebbe proprio ricondursi a quello. 
Siano $A, B$ due curve irriducibili di $F$, e supponiamo che il sistema lineare $|A+B|$ sia irriducibile ed infinito, quindi contenga qualche curva irriducibile $C$; indichiamo inoltre con $\alpha, \beta, \gamma$ i cicli a due dimensioni immagini di $A, B, C$ in $V$, orientati per ora in modo arbitrario. Tra $\alpha, \beta, \gamma$ intercederà certamente una delle omologie $\gamma= \pm \alpha \pm \beta$, ma non si potrà asserire ch'essa sia proprio la $\gamma=\alpha+\beta$ finchè ad $\alpha$ e $\beta$ non si attribuiscono le orientazioni che derivano per continuità da quella di $\gamma$, quando $C$, variando nel proprio sistema lineare, va a spezzarsi in $A+B$.

$\dot{\mathrm{E}}$ però facile vedere, che, se le orientazioni dei cicli $\alpha$ immagini di curve effettive $A$, si fissano in base al criterio uniforme del LeFscheTz $\left.{ }^{22}\right)$, secondo il quale quelle orientazioni restan assegnate in modo che (orientata opportunamente la $V$ ) il numero effettivo $(A B)$ delle intersezioni di due curve risulti eguale al numero algebrico $(\alpha \beta)$, allora quando sia $C \equiv A+B$ (o più in generale $C \equiv A+B$ ) è sempre $\gamma=\alpha+\beta$. Per persuadersene basta segare le curve considerate con una curva effettiva $D$ a cui corrisponda il ciclo $\delta$, e tener presente che $(A+B, D)=(A B)+(B D),(\alpha+\beta, \delta)=(\alpha \delta)+(\beta \delta)$.

E d'altronde solo in base a tal criterio che si puó parlare senz' ambiguità del ciclo di $V$ associato ad una curva riducibile di $F$.

Ciò premesso, suppongasi che la $A$, irriducibile, sia reale. Il ciclo $\alpha$ coinciderà materialmente col suo trasformato $\alpha^{\prime}$ mediante $S$; ma poichè $\alpha$ è riemanniana di $A$, la trasformazione indotta ivi da $S$ è inver'sa, onde, con riguardo alle orientazioni, sarà rigorosamente $\alpha^{\prime}=-\alpha$. In altre parole, secondo la nostra terminologia, $\alpha$ è un ciclo immaginar $i o$ puro della $V$.

La conclusione sussiste anche se $A$ è riducibile; e per persuadersene basta aggiungere ad $A$ una $B$ irriducibile e reale, ad es. la sezione di $F$ con una forma reale d'ordine abbastanza elevato, per guisa che nel sistema lineare $|A+B|$ esista qualche curva $C$ irriducibile e reale $\left({ }^{23}\right)$. Invero allora, col solito significato dei simboli, si ha $\gamma=\alpha+\beta, \beta^{\prime}=-\beta, \gamma^{\prime}=-\gamma, \gamma^{\prime}=\alpha^{\prime}+\beta^{\prime}$, quindi $\alpha^{\prime}=-\alpha$.

Invece se $A$ è qualunque (ma effettiva) in $V$, ed $\bar{A}$ è la curva coniugata, i due cicli $\alpha, \bar{\alpha}$ son trasformati materialmente uno nell' altro da $S$, però, con riguardo alle orientazioni, è $\alpha^{\prime}=-\bar{\alpha}$; bastando per ciò osservare che la curva $A+\bar{A}$ è reale, quindi il ciclo $\alpha+\bar{\alpha}$ è trasformato da $S$ nell' opposto,

(i2) Vedi la nota (ia).

(23) Un sistema lineare reale può non contenere curve reali, ma quando ne contiene una ne contiene infinite, tra cui ve n'è certo di irriducibili se lo è il sistema: e qù̀ una curva reale è la stessa $A+B$. In argomento, cfr. loco cit. (1), $\alpha$, n. 2. 
il che appunto si verifica quando $\alpha^{\prime}=-\bar{\alpha}$, non quando $\alpha^{\prime}=\bar{\alpha}$. In conclusione si ha dunque che:

Una curva reale della superficie $\mathrm{F}$ è rappresentata entro alla riemanniana $\mathrm{V}$ da un ciclo immaginario puro, e due cuvve coniugate son rappresentate da due cicli ciascuno dei quali è omologo all'opposto dell'altro.

10. Partiamoci ora da $p$ curve $C_{1}, C_{2}, \ldots, C_{\phi}$, costituenti una base (complessa) sulla superficie $F$. Per quanto non sia strettamente necessario, supponiamole scelte nella maniera più generale, quindi suscettibili di variare in sistemi lineari infiniti di dimensione virluale positiva, ecc.

Le curve reali $C_{1}+\bar{C}_{1}, C_{2}+\bar{C}_{2}, \ldots, C_{p}+\bar{C}_{p}$ non saranro, in generale, tutte algebricamente indipendenti, ma di tali se ne potranno trovare al più $l$, siano le prime, per guisa che ogni altra sarà algebricamente dipendente da esse. Posto $A_{i}=C_{i}+\bar{C}_{i}(i=1,2, \ldots, l)$, basta ripetere un ragionamento del $n .^{\circ} 7$ per dedurne che $A_{1}, A_{2}, \ldots, A_{l}$ dànno su $F$ una base reale (cioè per le curve reali) onde $l$ altro non è che il numero base reale ō.

In dettaglio, se $D$ è una curva (effettiva) reale di $F$, la relazlone

$$
\mu D \equiv \lambda_{1} C_{1}+\lambda_{2} C_{2}+\ldots+\lambda_{\rho} C_{\rho},
$$

che ne esprime la dipendenza dai $C_{t}$ sussiste assieme alla

$$
\mu D \equiv \lambda_{1} \bar{C}_{1}+\lambda_{2} \bar{C}_{2}+\ldots+\lambda_{p} \bar{C}_{\varphi}
$$

fra le curve coniugate, e dalle due, sommando, discende

$$
2 \mu D \equiv \lambda_{1}\left(C_{1}+\bar{C}_{1}\right)+\lambda_{2}\left(C_{2}+\bar{C}_{2}\right)+\ldots+\lambda_{\rho}\left(C_{\rho}+\bar{C}_{\rho}\right),
$$

donde, posto che tutte le $C_{j}+\bar{C}_{j}$ dipendono dalle $A_{i}$, si trae la conclusione.

Ora ripristiniamo su $F$ una base complessa includente le $A_{i}$ aggregando a queste certe $\sigma=\rho-\bar{\rho}$ curve $B_{1}, B_{2}, \ldots, B_{\sigma}$ scelte adeguatamente, ma per il resto nel modo più generale; ed indicate, al solito con $B_{1}, \ldots, \bar{B}_{\sigma}$ le coniugate, mettiamo in evidenza che le curve reali $B_{t}+\bar{B}_{t}$ dipendono dalle $A_{i}$ scrivendo le relazioni

$$
\mu_{t}\left(B_{t}+\bar{B}_{t}\right) \equiv \lambda_{t_{1}} A_{1}+\lambda_{t_{2}} A_{2}+\ldots+\lambda_{t \rho} A_{\rho}^{-}\left({ }^{24}\right), \quad(l=1,2, \ldots, \sigma)
$$

con $\mu_{t} \neq 0$. Passando ai corrispondenti cicli $\alpha_{i}, \beta_{t}, \bar{\beta}_{t}$ della $V$, avremo intanto,

(24) Per la maniera generale con cui furono scelti i $C_{j}, B_{t}$ è da escludersi che sia necessario aggiungere ai due membri una stessa curva $E_{3}$, che del resto nr.n darebbe aleun incomodo. 
perchè le $A_{i}$ son reali $\alpha_{i}^{\prime}=-\alpha_{i}$ ed inoltre, per quel che s'è visto $\beta_{t}^{\prime}=-\bar{\beta}_{t}$ onde dalle (14) si dedurranno le omologie

$$
\mu_{t}\left(\beta_{t}-\beta_{t}{ }^{\prime}\right)=\lambda_{t_{1}} \alpha_{1}+\lambda_{t_{2}} \alpha_{2}+\ldots+\lambda_{t_{1}} \alpha_{p}^{-},
$$

ed anche, dopo aver moltiplicato i due membri per 2 , e tenuto conto che $\alpha_{i}=-\alpha_{i}{ }^{\prime}$

$$
2 \mu_{t} \beta_{t}-\left(\lambda_{t_{1}} \alpha_{1}+\ldots+\lambda_{t_{p}} \alpha_{\rho}^{-}\right)=2 \mu_{t} \beta_{t}^{\prime}-\left(\lambda_{t_{1}} \alpha_{1}^{\prime}+\ldots+\lambda_{t_{p}^{-}} \alpha_{\rho}^{\prime}\right) .
$$

Queste vengono a dire che $\mathrm{i} \sigma$ cicli algebrici

$$
\gamma_{t}=2 \mu_{t} \beta_{t}-\left(\lambda_{t_{1}} \alpha_{1}+\ldots+\lambda_{t_{p}} \alpha_{\rho}^{-}\right), \quad(t=1,2, \ldots, \sigma)
$$

son trasformati in cicli omologhi da $S$, vale a dire son reali; e d'altronde, come mostrano le (17), essi posson sostituirsi ai $\beta_{t}$, cioè presi assieme agli $\alpha_{i}$ dànno un sistema di $\rho$ cicli algebrici indipendenti, su cui la simmetria $S$ opera colla sostituzione

$$
\alpha_{i}^{\prime}=-\alpha_{i}, \quad \gamma_{t}^{\prime}=\gamma_{t}\left({ }^{25}\right),(i=1,2, \ldots \bar{\rho} ; t=1,2, \ldots, \sigma) .
$$

Ogni ciclo algebrico $\delta$ è pertanto dipendente dagli $\alpha_{t}, \gamma_{t}$, cioè legato ad essi da un'omologia

$$
v \delta=\lambda_{1} \alpha_{1}+\ldots+\lambda_{i}^{-} \alpha_{\beta}^{-}+\mu_{1} \gamma_{1}+\ldots+\mu_{\sigma} \gamma_{\sigma},
$$

$(v \neq 0)$ che, trasformata mediante $S$, dà, a norma delle (18) l'altra

$$
v \delta^{\prime}=-\lambda_{1} \alpha_{1}-\ldots-\lambda_{\rho}^{-} \alpha_{\rho}^{-}+\mu_{1} \gamma_{1}+\ldots+\mu_{\sigma} \gamma_{\sigma}
$$

ed infine sommando porge

$$
\nu\left(\delta+\delta^{\prime}\right)=2 \mu_{1} \gamma_{1}+2 \mu_{2} \gamma_{2}+\ldots+2 \mu_{\sigma} \gamma_{\sigma} .
$$

In particolare se $\delta$ è reale si ha $\delta=\delta^{\prime}$ ed allora la (19) dimostra che $\delta$ dipende dai $\gamma_{t}$. Resta cosi provato che ogni ciclo algebrico reale $d i \mathrm{~V} d i$ pende dagli analoghi cicli indipendenti $\gamma_{1}, \gamma_{2}, \ldots, \gamma_{\sigma}$, quindi che $i$ cicli algebrici portano al numero $h$ contributo $\sigma=\rho-\tilde{\rho}$, c. d. d.

Termineremo notando che l'invarianza assoluta del carattere $\tau=$ $=\frac{1}{2}\left(R_{2}-Z\right)+\rho-\bar{\rho}$ (nel campo reale) puó in generale desumersi, oltrechè dal suo significato, anche dal valutare l'influenza che l'introduzione di curve

(25) Si noti che ad un ciclo algebrico reale $\gamma\left(\gamma^{\prime}=\gamma\right)$ non può corvispondere su $\mathrm{F}$ una curva effettiva $\Gamma$. Difatti detta $\bar{\gamma}$ la coniugata, sarebbe $\gamma^{\prime}=-\bar{\gamma}$, quindi $\gamma+\bar{\gamma}=0$ ed allora alla curva effettiva $\mathrm{T}+\bar{\Gamma}$ corrisponderebbe un ciclo nullo, il che è assurdo (LeFscheтz, loco cit. ("), Cap. II, n. 6). Invece ad una curva virtuale come la $A-\bar{A}$ corrisponde effettivamente un ciclo $\alpha-\bar{x}=\alpha+x^{\prime}$ reatle. 
eccezionali di $1^{\text {a }}$ specie ha sulla relativa espressione; bastando, allo scopo, osservare, che per l'intervento d'una (nuova) curva eccezionale reale, tutti i numeri $R_{2}, Z, \rho, \bar{\rho}$ aumentano d'una unità; mentre l'introduzione di due curve eccezionali immaginarie coniugate non altera $Z$, produce in $\bar{\rho}$ l'aumento d' una unità ed in $R_{2}$, $\rho$ quello di due unità.

\section{§ 5. Esempî e controlli.}

11. Per $\mathbf{i}$ varî casi relativi alle superficie razionali, rinviamo ai lavori ricordati. Qui esamineremo i seguenti casi nuovi :

a) Rigate ivazionali di genere $q .-\mathrm{Ci}$ riferiremo ad una rigata reale $F$ 'd'uno spazio $S_{n}$ priva di curve eccezionali di $1^{\text {a }}$ specie (e di punti multipli). Il relativo genere geometrico è nullo, quindi si deve avere $Z=R_{2}-2(\rho-\bar{\rho})$.

Poichè su $F$ la base è costituita da una generatrice e da una sezione iperpiana, entrambe curve reali $\left({ }^{26}\right)$, cosi si ha $\rho=\bar{p}=2$; d'altronde $I=-4 q$, $R_{\mathrm{z}}=I+4 q+2=2$, e pertanto secondo la formula dev' essere $Z=2$.

Di fatto la $F$ consta d' un certo numero $\mu \geq 0$ di falde (eguale al numero dei rami della sezione iperpiana) ciascuna delle quali ha la connessione d' una quadrica rigata, vale a dire 2 . E tal valore ha pure la connessione totale $Z$ come risulta dall' espressione che la definisce (cfr, anche $\left({ }^{16}\right)$ ).

b) Superficie delle coppie ordinate di punti d'una curva reale $C$ di genere $p(>0)$. - D' una tal superficie si possono considerare due modelli reali $\Phi, \Phi^{\prime}$, distinti per trasformazioni birazionali reali, e legati alle simmetrie $\left({ }^{27}\right)(P, Q) \rightarrow(\bar{Q}, \bar{P}),(P, Q) \rightarrow(\bar{P}, \bar{Q})$.

Nel citato lavoro abbiamo trovato, mediante investigazione diretta, che $Z=2 p$ per la $\Phi$, e $Z=2$ per la $\Phi^{\prime}$. Verifichiamo con tali valori la formula $Z=R_{2}-2 h$, determinando il valore di $h$ ed attribuendo ad $R_{z}$ il valore noto $4 p^{2}+2\left({ }^{28}\right)$; dovremo trovare nei due casi $h=2 p^{2}-p+1, h=2 p^{2}$.

Ove poi si tenga presente, che, supposta la $C$ a moduli generali, si ha $\rho=2\left({ }^{29}\right)$, mentre $\bar{\rho}$ vale rispettivamente 1 e 2 in quanto nel primo caso $\mathrm{i}$

${ }^{(26)}$ La generatrice può anche non esser reale, ma appartiene ad un sistema algebrico reale, il che è equivalente.

(77) Vedi il lavoro citato (5), n. 5 .

${ }^{(28)}$ Vedi, anche per $\mathrm{i}$ eicli $\left({ }^{20}\right)$, Lesfschetz, loco cit. $\left({ }^{2}\right)$, Cap. III, § VII.

(29) F. SEverI, Sulle corrispondenze fra $i$ punti d'una curva algebrica, ecc. [Memorie della R. Acc. di Torino, 64 (1903), pp. 1-49], Parte $2^{\text {a }}$. 
due fasci unisecanti sono immaginarî coniugati, nel secondo reali, si vede che risulterà rispettivamente $\tau=2 p^{2}-p, \tau=2 p^{2}$, di guisa che, come avvertito, i valori di $\tau$ saranno affatto diversi.

Designata con $R$ la riemanniana di $C$, con $\gamma_{i}, \delta_{i}(i=1,2, \ldots, 2 p)$ due sistemi di cicli indipendenti (ma non necessariamente primitivi) di $R$, e con $P, Q$ due punti ivi fissati, si possono considerare in $V$ gli $R_{z}$ eicli a due dimensioni indipendenti indicati dalle

$$
M=(P, R), \quad N=(R, Q), \quad \Gamma_{i k}=\left(\gamma_{i}, \delta_{k}\right), \quad(i, k=1,2, \ldots, 2 p),
$$

con o,vrio significato dei simboli.

Orientata la $R$, lo sono senz'altro i cicli $M, N$; le orientazioni dei $\mathrm{\Gamma}_{i k}$ si fisseranno nel modo seguente: Scelto su $\gamma_{i}$ un punto $H_{i}$, e su $\delta_{k}$ un punto $K_{k}$, si considerino su $\Gamma_{i k}$ i due cicli (orientati perchè lo sono i $\left.\gamma_{i}, \delta_{k}\right) c_{i}=\left(\gamma_{i}, K_{k}\right)$, $d_{k}=\left(H_{i}, \delta_{k}\right)$ che s'incontrano nel punto $\left(H_{i}, K_{k}\right)$, indi si orienti $\Gamma_{i k}$ in modo che quel punto porti al numero algebrico $\left(c_{i}, d_{k}\right)$ contributo +1 .

A questo punto conviene procedere separatamente:

$b_{1}$ ) Primo modello. Scelti come $\delta_{i}$ i coniugati dei $\gamma_{i}$, si vede facilmente, tenendo conto del modo come vennero fissate le orientazioni, che la simmetria $S$ di $V$ produce sui cicli (20) la sostituzione

$$
M^{\prime}=-N, \quad N^{\prime}=-M, \quad \Gamma_{i k}^{\prime}=-\Gamma_{k i},
$$

onde si ottengono subito i cicli reali indipendenti

$$
M-N, \quad \Gamma_{i k}-\Gamma_{k i} \quad(i<k),
$$

e coll' aiuto delle (21) si trova che ogni altro ciclo reale a due dimensioni di $V$ dipende da essi. Dunque $h=1+\left(\begin{array}{c}2 p \\ 2\end{array}\right)=2 p^{2}-p+1$ come previsto.

$\mathrm{b}_{2}$ ) Secondo modello. Si scelgano i $\gamma_{i}$ in modo che la simmetria $s$ di $R$ immagine del coniugio di $C$ produca su essi la sostituzione

$$
\gamma_{i}^{\prime}=\gamma_{i}, \quad \gamma_{j}^{\prime}=-\gamma_{j}\left({ }^{30}\right), \quad(i=1,2, \ldots, p ; j=p+1, \ldots, 2 p)
$$

ed i $\delta_{i}$ identici ai $\gamma_{i}$; allora si trova che la sostituzione indotta da $S$ sui cicli $(20)$ è

$$
M^{\prime}=-M, \quad N^{\prime}=-N, \quad \Gamma_{i k}^{\prime}=\Gamma_{i k}, \quad \Gamma_{r s}^{\prime}=-\Gamma_{r s},
$$

dove gl'indici $i, k$ assumono valori entrambi non maggiori o maggiori di $p$,

(30) Cfr. loco cit. $\left({ }^{19}\right)$, Memoria I, prime righe del n. 3. Qui nulla importa aver cicli non primitivi. 
e gl' indici $r$, s i rimanenti. Ne viene che stavolta il sistema fondamentale per $i$ cicli algebrici reali è dato dai $\Gamma_{i k}$, talchè, conformemente alla previsione, si ha $h=2 p^{z}$.

c) Superficie delle coppie non ordinate di punti della curva precedente. - Se la. $C$ è a moduli generali, si ha un solo modello reale $\Psi$ corrispondente alla simmetria $(P, Q) \rightarrow(\bar{P}, \bar{Q})$, che supporremo privo di curve eccezionali. La connessione totale $Z$ della sua parte reale, determinata direttamente nel lavoro testè ricordato, ha il valore $p+1$.

Per la nostra $\Psi$ si ha $I=2 p^{2}-5 p-1\left({ }^{31}\right), q=p$, quindi $R_{2}=2 p^{2}-p+1$; inoltre $\rho=\bar{\rho}=1$, onde si dovrà trovare $h=\tau=2\left(\begin{array}{l}p \\ 2\end{array}\right)$.

Scelti come in $\left.b_{2}\right)$ i $\gamma_{i}, \delta_{i}$, si hanno adesso in $V$ gli $R_{2}=1+\left(\begin{array}{c}2 p \\ 2\end{array}\right)$ cicli a due dimensioni indipendenti indicati dai simboli

$$
M=(P, R), \quad \Gamma_{i k}=\left(\gamma_{i}, \gamma_{k}\right)
$$

dove ad $i, k$ si diano $\mathrm{i}$ valori delle combinazioni binarie degli elementi 1 , $2, \ldots, 2 p$; e la sostituzione su essi indotta da $S$ è

$$
M^{\prime}=-M, \quad \Gamma_{i k}^{\prime}=\Gamma_{i k}, \quad \Gamma_{r s}^{\prime}=-\Gamma_{r s},
$$

gl' indici variando come nelle (22) (coll'avvertenza che ora $\Gamma_{i k}=\Gamma_{k i}$ ). Il sistema fondamentale per $i$ cicli algebrici reali è dato dai $\Gamma_{i k}$, che sono appunto, come previsto, in numero di $2\left(\begin{array}{l}p \\ 2\end{array}\right)$.

Padova, febbraio 1928.

(31) F. SEVERI, Sulle superficie che rappresentano le coppie di punti d' una curva algebrica. [Atti della R. Ace. di Torino, 38 (1908)], n. 6. 